DOI 10.7603/s40956-016-0006-0

Moroccan J. Pure and Appl. Anal.(MJPAA)

RESEARCH ARTICLE

Volume 2(2), 2016, Pages 79-90

ISSN: $2351-8227$

\title{
On a $(p, k)$-analogue of the Gamma function and some associated Inequalities
}

\author{
K. Nantomah ${ }^{a}$, E. Prempeh ${ }^{b}$ And S. B. Twum ${ }^{a}$
}

\begin{abstract}
In this paper, we introduce a new two-parameter deformation of the classical Gamma function, which we call a $(p, k)$-analogue of the Gamma function. We also provide some identities generalizing those satisfied by the classical Gamma function. Furthermore, we establish some inequalities involving this new function.
\end{abstract}

2010 Mathematics Subject Classification.33B15, 26D07, 26D15.

Key words and phrases. Gamma function, $(p, k)$-analogue, inequality.

\section{Introduction}

The classical Euler's Gamma function, $\Gamma(x)$ is usually defined for $x>0$ by

$$
\Gamma(x)=\int_{0}^{\infty} t^{x-1} e^{-t} d t=\lim _{n \rightarrow \infty} \frac{n ! n^{x}}{x(x+1)(x+2) \ldots(x+n)}
$$

It is well-known that $\Gamma(x)$ satisfies the following basic relations.

$$
\begin{aligned}
& \Gamma(n+1)=n !, \quad n \in \mathbb{Z}^{+} \cup\{0\}, \\
& \Gamma(x+1)=x \Gamma(x), \quad x \in \mathbb{R}^{+} .
\end{aligned}
$$

Received February 20, 2016 - Accepted June 08, 2016.

(C) The Author(s) 2016. This article is published with open access by Sidi Mohamed Ben Abdallah University

${ }^{a}$ Department of Mathematics, University for Development Studies, Navrongo Campus, P. O. Box 24, Navrongo, UE/R, Ghana.

e-mail: mykwarasoft@yahoo.com, knantomah@uds.edu.gh

stwum@uds.edu.gh

${ }^{b}$ Department of Mathematics, Kwame Nkrumah University of Science and Technology, Kumasi, Ghana.

e-mail: eprempeh.cos@knust.edu.gh 
Closely associated with the Gamma function is the Digamma or Psi function $\psi(x)$, which is defined for $x>0$ as the logarithmic derivative of the Gamma function. That is,

$$
\begin{aligned}
\psi(x) & =\frac{d}{d x} \ln \Gamma(x)=\frac{\Gamma^{\prime}(x)}{\Gamma(x)}, \\
& =-\gamma+(x-1) \sum_{n=0}^{\infty} \frac{1}{(n+1)(n+x)}, \\
& =-\gamma-\frac{1}{x}+\sum_{n=1}^{\infty} \frac{x}{n(n+x)}
\end{aligned}
$$

where $\gamma=\lim _{n \rightarrow \infty}\left(\sum_{k=1}^{n} \frac{1}{k}-\ln n\right)=0.577215664 \ldots$ is the Euler-Mascheroni's constant. The Polygamma functions, $\psi^{(m)}(x)$ are defined for $x>0$ and $m \in \mathbb{N}$ as

$$
\begin{aligned}
\psi^{(m)}(x) & =\frac{d^{m}}{d x^{m}} \psi(x)=\frac{d^{m+1}}{d x^{m+1}} \ln \Gamma(x) \\
& =(-1)^{m+1} m ! \sum_{n=0}^{\infty} \frac{1}{(n+x)^{m+1}}
\end{aligned}
$$

where $\psi^{(0)}(x) \equiv \psi(x)$.

The $p$-analogue (also known as $p$-extension or $p$-deformation) of the Gamma function is defined for $p \in \mathbb{N}$ and $x>0$ as

$$
\Gamma_{p}(x)=\frac{p ! p^{x}}{x(x+1) \ldots(x+p)}
$$

where $\lim _{p \rightarrow \infty} \Gamma_{p}(x)=\Gamma(x)$. See [1, p. 270]. It satisfies the identities:

$$
\begin{aligned}
\Gamma_{p}(x+1) & =\frac{p x}{x+p+1} \Gamma_{p}(x), \\
\Gamma_{p}(1) & =\frac{p}{p+1} .
\end{aligned}
$$

The $p$-analogues of the Digamma and Polygamma functions are defined for $x>0$ as

$$
\begin{aligned}
\psi_{p}(x) & =\frac{d}{d x} \ln \Gamma_{p}(x)=\ln p-\sum_{n=0}^{p} \frac{1}{n+x} \\
\psi_{p}^{(m)}(x) & =\frac{d^{m}}{d x^{m}} \psi_{p}(x)=(-1)^{m-1} m ! \sum_{n=0}^{p} \frac{1}{(n+x)^{m+1}}
\end{aligned}
$$

where $\psi_{p}^{(0)}(x) \equiv \psi_{p}(x)$. 
In 2007, Díaz and Pariguan [2] also defined the $k$-analogue of the Gamma function for $k>0$ and $x \in \mathbb{C} \backslash k \mathbb{Z}^{-}$as

$$
\Gamma_{k}(x)=\int_{0}^{\infty} t^{x-1} e^{-\frac{t^{k}}{k}} d t=\lim _{n \rightarrow \infty} \frac{n ! k^{n}(n k)^{\frac{x}{k}-1}}{(x)_{n, k}}
$$

where $\lim _{k \rightarrow 1} \Gamma_{k}(x)=\Gamma(x)$ and $(x)_{n, k}=x(x+k)(x+2 k) \ldots(x+(n-1) k)$ is the Pochhammer $k$-symbol. The $k$-analogue also satisfies the identities:

$$
\begin{aligned}
\Gamma_{k}(x+k) & =x \Gamma_{k}(x), \quad x \in R^{+} \\
\Gamma_{k}(k) & =1
\end{aligned}
$$

Similarly, the $k$-analogues of the Digamma and Polygamma functions are defined for $x>0$ as

$$
\begin{aligned}
\psi_{k}(x) & =\frac{d}{d x} \ln \Gamma_{k}(x)=\frac{\ln k-\gamma}{k}-\frac{1}{x}+\sum_{n=1}^{\infty} \frac{x}{n k(n k+x)}, \\
\psi_{k}^{(m)}(x) & =\frac{d^{m}}{d x^{m}} \psi_{k}(x)=(-1)^{m+1} m ! \sum_{n=0}^{\infty} \frac{1}{(n k+x)^{m+1}}
\end{aligned}
$$

where $\psi_{k}^{(0)}(x) \equiv \psi_{k}(x)$.

The purpose of this paper is to introduce a new two-parameter deformation of the classical Gamma function, called a $(p, k)$-analogue of the Gamma function. In addition, we provide some identities and inequalities involving this function. We present our results in the following section.

\section{Results and Discussion}

Definition 2.1. Let $p \in \mathbb{N}$ and $k>0$. Then the $(p, k)$-analogue (also called the $(p, k)$-deformation or $(p, k)$-generalization) of the Gamma function is defined as

$$
\begin{aligned}
\Gamma_{p, k}(x) & =\int_{0}^{p} t^{x-1}\left(1-\frac{t^{k}}{p k}\right)^{p} d t \\
& =\frac{(p+1) ! k^{p+1}(p k)^{\frac{x}{k}-1}}{x(x+k)(x+2 k) \ldots(x+p k)}
\end{aligned}
$$

for $x \in \mathbb{R}^{+}$. It satisfies the identities:

$$
\begin{aligned}
\Gamma_{p, k}(x+k) & =\frac{p k x}{x+p k+k} \Gamma_{p, k}(x), \\
\Gamma_{p, k}(a k) & =\frac{p+1}{p} k^{a-1} \Gamma_{p}(a), \quad a \in \mathbb{R}^{+} \\
\Gamma_{p, k}(k) & =1 .
\end{aligned}
$$


Also, observe that $\Gamma_{p, k}(x)$ satisfies the following commutative diagram.

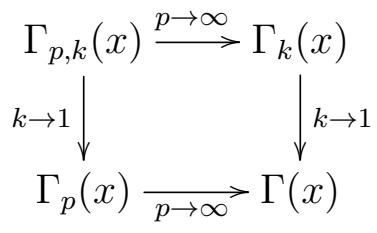

The $(p, k)$-analogue of the Digamma function is defined as the logarithmic derivative of $\Gamma_{p, k}(x)$. That is

$$
\psi_{p, k}(x)=\frac{d}{d x} \ln \Gamma_{p, k}(x)=\frac{\Gamma_{p, k}^{\prime}(x)}{\Gamma_{p, k}(x)} .
$$

The function $\psi_{p, k}(x)$ satisfies the following series and integral representations.

$$
\begin{aligned}
\psi_{p, k}(x) & =\frac{1}{k} \ln (p k)-\sum_{n=0}^{p} \frac{1}{(n k+x)} \\
& =\frac{1}{k} \ln (p k)-\int_{0}^{\infty} \frac{1-e^{-k(p+1) t}}{1-e^{-k t}} e^{-x t} d t
\end{aligned}
$$

The $(p, k)$-analogue of the Polygamma functions are defined as

$$
\begin{aligned}
\psi_{p, k}^{(m)}(x)=\frac{d^{m}}{d x^{m}} \psi_{p, k}(x) & =(-1)^{m+1} m ! \sum_{n=0}^{p} \frac{1}{(n k+x)^{m+1}} \\
& =(-1)^{m+1} \int_{0}^{\infty}\left(\frac{1-e^{-k(p+1) t}}{1-e^{-k t}}\right) t^{m} e^{-x t} d t
\end{aligned}
$$

for $m \in \mathbb{N}$, where $\psi_{p, k}^{(0)}(x) \equiv \psi_{p, k}(x)$. It follows easily from (8) that,

$$
\psi_{p, k}^{(m)}(x)= \begin{cases}>0 & \text { if } m \text { is odd } \\ <0 & \text { if } m \text { is even }\end{cases}
$$

which means that the function $\psi_{p, k}^{\prime}(x)$ is a completely monotonic function of $x$, for $x \in \mathbb{R}^{+}$.

Remark 2.1. From the identity (3), we obtain the relations

$$
\begin{aligned}
\psi_{p, k}(x+k)-\psi_{p, k}(x) & =\frac{1}{x}-\frac{1}{x+p k+k}, \\
\psi_{p, k}^{(m)}(x+k)-\psi_{p, k}^{(m)}(x) & =\frac{(-1)^{m} m !}{x^{m+1}}-\frac{(-1)^{m} m !}{(x+p k+k)^{m+1}}, \quad m \in \mathbb{N} .
\end{aligned}
$$

Also from (6), we obtain the relation

$$
\psi_{p, k}(k)=\frac{1}{k}[\ln (p k)-H(p+1)]
$$

where $H(n)$ is the nth harmonic number. 
The $(p, k)$-analogue of the classical Beta function is defined as

$$
B_{p, k}(x, y)=\frac{\Gamma_{p, k}(x) \Gamma_{p, k}(y)}{\Gamma_{p, k}(x+y)}, \quad x>0, y>0 .
$$

Lemma 2.1. The function $\psi_{p, k}(x)$ satisfies the following limit properties.

(i) $\psi_{p, k}(x) \rightarrow \psi_{k}(x)$ as $p \rightarrow \infty$,

(ii) $\psi_{p, k}(x) \rightarrow \psi_{p}(x)$ as $k \rightarrow 1$,

(iii) $\psi_{p, k}(x) \rightarrow \psi(x)$ as $p \rightarrow \infty$ and $k \rightarrow 1$.

Proof. (i) By (6), we have

$$
\begin{aligned}
\lim _{p \rightarrow \infty} \psi_{p, k}(x) & =\lim _{p \rightarrow \infty}\left[\frac{1}{k} \ln (p k)-\frac{1}{x}-\sum_{n=1}^{p} \frac{1}{(n k+x)}-\sum_{n=1}^{p} \frac{1}{n k}+\sum_{n=1}^{p} \frac{1}{n k}\right] \\
& =\lim _{p \rightarrow \infty}\left[\frac{1}{k} \ln (p k)-\sum_{n=1}^{p} \frac{1}{n k}-\frac{1}{x}+\sum_{n=1}^{p} \frac{1}{n k}-\sum_{n=1}^{p} \frac{1}{(n k+x)}\right] \\
& =\lim _{p \rightarrow \infty}\left[\frac{1}{k} \ln k+\frac{1}{k} \ln p-\frac{1}{k} \sum_{n=1}^{p} \frac{1}{n}-\frac{1}{x}+\sum_{n=1}^{p} \frac{x}{n k(n k+x)}\right] \\
& =\frac{1}{k} \ln k+\frac{1}{k} \lim _{p \rightarrow \infty}\left[\ln p-\sum_{n=1}^{p} \frac{1}{n}\right]-\frac{1}{x}+\sum_{n=1}^{\infty} \frac{x}{n k(n k+x)} \\
& =\frac{1}{k} \ln k-\frac{\gamma}{k}-\frac{1}{x}+\sum_{n=1}^{\infty} \frac{x}{n k(n k+x)} \\
& =\psi_{k}(x) .
\end{aligned}
$$

(ii) Also by (6), we have

$$
\lim _{k \rightarrow 1} \psi_{p, k}(x)=\lim _{k \rightarrow 1}\left[\frac{1}{k} \ln (p k)-\sum_{n=0}^{p} \frac{1}{(n k+x)}\right]=\ln p-\sum_{n=0}^{p} \frac{1}{(n+x)}=\psi_{p}(x) .
$$

(iii) This follows easily from (i) or (ii). That is,

$$
\begin{aligned}
\lim _{k \rightarrow 1}\left(\lim _{p \rightarrow \infty} \psi_{p, k}(x)\right) & =\lim _{k \rightarrow 1} \psi_{k}(x)=\psi(x), \quad \text { or } \\
\lim _{p \rightarrow \infty}\left(\lim _{k \rightarrow 1} \psi_{p, k}(x)\right) & =\lim _{p \rightarrow \infty} \psi_{p}(x)=\psi(x) .
\end{aligned}
$$

Lemma 2.2. Let $\gamma_{p, k}=-\psi_{p, k}(1)$ be the $(p, k)$-analogue of the Euler-Mascheroni's constant. Then $\gamma_{p, k} \rightarrow \gamma$ as $p \rightarrow \infty$ and $k \rightarrow 1$.

Proof. Proceed as follows

$$
\lim _{k \rightarrow 1} \psi_{p, k}(1)=\lim _{k \rightarrow 1}\left[\ln (p k)-\sum_{n=0}^{p} \frac{1}{(n k+1)}\right]
$$




$$
=\ln p-\sum_{n=0}^{p} \frac{1}{n+1} \text {. }
$$

Then,

$$
\begin{aligned}
\lim _{p \rightarrow \infty}\left(\lim _{k \rightarrow 1} \gamma_{p, k}\right) & =\lim _{p \rightarrow \infty}\left(-\lim _{k \rightarrow 1} \psi_{p, k}(1)\right) \\
& =-\lim _{p \rightarrow \infty}\left[\ln p-\sum_{n=0}^{p} \frac{1}{n+1}\right] \\
& =-\lim _{p \rightarrow \infty}\left[\ln p-\sum_{n=1}^{p} \frac{1}{n}+\sum_{n=1}^{p} \frac{1}{n}-1-\sum_{n=1}^{p} \frac{1}{n+1}\right] \\
& =-\lim _{p \rightarrow \infty}\left[\ln p-\sum_{n=1}^{p} \frac{1}{n}\right]+1-\sum_{n=1}^{\infty}\left[\frac{1}{n}-\frac{1}{n+1}\right] \\
& =\gamma .
\end{aligned}
$$

Definition 2.2. A function $f$ is said to be logarithmically convex if the following inequality holds for all $x, y>0$.

$$
\log f(\alpha x+\beta y) \leq \alpha \log f(x)+\beta \log f(y)
$$

or equivalently

$$
f(\alpha x+\beta y) \leq(f(x))^{\alpha}(f(y))^{\beta}
$$

where $\alpha, \beta>0$ such that $\alpha+\beta=1$.

Theorem 2.1. The function, $\Gamma_{p, k}(x)$ is logarithmically convex.

Proof. We want to show that for $x, y>0$ and $\alpha, \beta>0$ such that $\alpha+\beta=1$,

$$
\Gamma_{p, k}(\alpha x+\beta y) \leq\left(\Gamma_{p, k}(x)\right)^{\alpha}\left(\Gamma_{p, k}(y)\right)^{\beta} .
$$

Recall that the Young's inequality is given by

$$
x^{\alpha} y^{\beta} \leq \alpha x+\beta y
$$

where $x, y>0$ and $\alpha, \beta>0$ such that $\alpha+\beta=1$. By this, we obtain

$$
\left(k+\frac{x}{t}\right)^{\alpha}\left(k+\frac{y}{t}\right)^{\beta} \leq k+\frac{\alpha x+\beta y}{t} .
$$

Next, taking $\prod_{t=1}^{p}$ on (15), we have

$$
\prod_{t=1}^{p}\left(k+\frac{x}{t}\right)^{\alpha}\left(k+\frac{y}{t}\right)^{\beta} \leq \prod_{t=1}^{p}\left(k+\frac{\alpha x+\beta y}{t}\right)
$$

which implies

$$
\left(\frac{(x+k)(x+2 k) \ldots(x+p k)}{1 \times 2 \times \cdots \times p}\right)^{\alpha}\left(\frac{(y+k)(y+2 k) \ldots(y+p k)}{1 \times 2 \times \cdots \times p}\right)^{\beta}
$$




$$
\leq \frac{(\alpha x+\beta y+k)(\alpha x+\beta y+2 k) \ldots(\alpha x+\beta y+p k)}{1 \times 2 \times \cdots \times p}
$$

which further implies

$$
\begin{aligned}
& \frac{p !}{(\alpha x+\beta y+k)(\alpha x+\beta y+2 k) \ldots(\alpha x+\beta y+p k)} \\
& \quad \leq\left(\frac{p !}{(x+k)(x+2 k) \ldots(x+p k)}\right)^{\alpha}\left(\frac{p !}{(y+k)(y+2 k) \ldots(y+p k)}\right)^{\beta} .
\end{aligned}
$$

Then, by multiplying (16) by the identities:

$$
\begin{aligned}
\frac{1}{\alpha x+\beta y} & \leq \frac{1}{x^{\alpha} y^{\beta}}, \\
(p+1) & =(p+1)^{\alpha+\beta}, \\
k^{p+1} & =\left(k^{p+1}\right)^{\alpha+\beta}, \\
(p k)^{\frac{\alpha x+\beta y}{k}}-1 & =(p k)^{\frac{\alpha x}{k}-\alpha}(p k)^{\frac{\beta y}{k}-\beta}
\end{aligned}
$$

we obtain

$$
\begin{aligned}
& \frac{(p+1) ! k^{p+1}(p k)^{\frac{\alpha x+\beta y}{k}-1}}{(\alpha x+\beta y)(\alpha x+\beta y+k)(\alpha x+\beta y+2 k) \ldots(\alpha x+\beta y+p k)} \\
& \leq\left(\frac{(p+1) ! k^{p+1}(p k)^{\frac{x}{k}-1}}{x(x+k)(x+2 k) \ldots(x+p k)}\right)^{\alpha}\left(\frac{(p+1) ! k^{p+1}(p k)^{\frac{y}{k}-1}}{y(y+k)(y+2 k) \ldots(y+p k)}\right)^{\beta}
\end{aligned}
$$

which is (13). That completes the proof.

Remark 2.2. Alternatively, a compact proof of Theorem 2.1 could have been as follows. By using the defintion of $\psi_{p, k}(x)$ and the fact that $\psi_{p, k}^{\prime}(x)>0$, it follows immediately that $\Gamma_{p, k}(x)$ is logarithmically convex. Then, from the definition 2.2 for $x, y>0$, $\alpha, \beta>0$ such that $\alpha+\beta=1$, we obtain the inequality (13).

Corollary 2.1. Let $p \in \mathbb{N}$ and $k>0$. Then the inequality

$$
\Gamma_{p, k}\left(\frac{x+y}{2}\right) \leq \sqrt{\Gamma_{p, k}(x) \Gamma_{p, k}(y)}
$$

holds for $x, y>0$.

Proof. This follows directly from Theorem 2.1 by letting $\alpha=\beta=\frac{1}{2}$.

Theorem 2.2. Let $p \in \mathbb{N}$ and $k>0$. Then the inequality

$$
\Gamma_{p, k}(n x) \leq(p k)^{\frac{x}{k}(n-1)} \Gamma_{p, k}(x)
$$

holds for $n \in \mathbb{N}$ and $x>0$.

Proof. It follows easily from (2) that

$$
\frac{\Gamma_{p, k}(n x)}{\Gamma_{p, k}(x)}=(p k)^{\frac{x}{k}(n-1)} \frac{x(x+k)(x+2 k) \ldots(x+p k)}{n x(n x+k)(n x+2 k) \ldots(n x+p k)}
$$

which completes the proof. 
Corollary 2.2. Let $p \in \mathbb{N}$ and $k>0$. Then the inequality

$$
\Gamma_{p, k}(x+y) \leq(p k)^{\frac{x+y}{2 k}} \sqrt{\Gamma_{p, k}(x) \Gamma_{p, k}(y)}
$$

holds for $x, y>0$.

Proof. From (17), and by using (18) for $n=2$, we obtain

$$
\begin{aligned}
\Gamma_{p, k}(x+y) & \leq \sqrt{\Gamma_{p, k}(2 x) \Gamma_{p, k}(2 y)} \\
& \leq(p k)^{\frac{x+y}{2 k}} \sqrt{\Gamma_{p, k}(x) \Gamma_{p, k}(y)} .
\end{aligned}
$$

Remark 2.3. Results similar to (13), (17), (18) and (19) for the (q, k)-analogue of the Gamma function can also be found in [3].

Lemma $2.3([9])$. Let $f:(0, \infty) \rightarrow(0, \infty)$ be a differentiable, logarithmically convex function. Then the function

$$
g(x)=\frac{(f(x))^{\alpha}}{f(\alpha x)}, \quad \alpha \geq 1
$$

is decreasing on its domain.

Theorem 2.3. Let $p \in \mathbb{N}, k>0$ and $\alpha \geq 1$. Then the inequality

$$
\frac{\left[\Gamma_{p, k}(y)\right]^{\alpha}}{\Gamma_{p, k}(\alpha y)} \leq \frac{\left[\Gamma_{p, k}(x)\right]^{\alpha}}{\Gamma_{p, k}(\alpha x)} \leq \frac{p}{p+1} k^{1-\alpha} \frac{1}{\Gamma_{p}(\alpha)}
$$

is valid for $k \leq x \leq y$.

Proof. Recall from Theorem 2.1 that $\Gamma_{p, k}(x)$ is logarithmically convex. Then by Lemma 2.3, the function $H(x)=\frac{\left[\Gamma_{p, k}(x)\right]^{\alpha}}{\Gamma_{p, k}(\alpha x)}$ is decreasing. Hence for $k \leq x \leq y$, we have $H(y) \leq H(x) \leq H(k)$ yielding the result $(20)$.

Theorem 2.4. Let $p \in \mathbb{N}, k>0$ and $\alpha \geq 1$. Then the inequality

$$
\frac{\left[\Gamma_{p, k}(1+k)\right]^{\alpha}}{\Gamma_{p, k}(\alpha+k)} \leq \frac{\left[\Gamma_{p, k}(x+k)\right]^{\alpha}}{\Gamma_{p, k}(\alpha x+k)} \leq 1
$$

is valid for $x \in[0,1]$.

Proof. Define $Q$ by $Q(x)=\frac{\left[\Gamma_{p, k}(x+k)\right]^{\alpha}}{\Gamma_{p, k}(\alpha x+k)}$ for $p \in \mathbb{N}, k>0$ and $\alpha \geq 1$. Let $\lambda(x)=\ln Q(x)$. Then

$$
\begin{aligned}
\lambda^{\prime}(x) & =\alpha \frac{\Gamma_{p, k}^{\prime}(x+k)}{\Gamma_{p, k}(x+k)}-\alpha \frac{\Gamma_{p, k}^{\prime}(\alpha x+k)}{\Gamma_{p, k}(\alpha x+k)} \\
& =\alpha\left[\psi_{p, k}(x+k)-\psi_{p, k}(\alpha x+k)\right] \\
& \leq 0
\end{aligned}
$$

since $\psi_{p, k}(x)$ is increasing for $x>0$. Hence $Q(x)$ is decreasing on $[0, \infty)$. Then for $x \in[0,1]$, we obtain $Q(1) \leq Q(x) \leq Q(0)$ yielding the result $(21)$. 
Remark 2.4. By letting $p \rightarrow \infty$ as $k \rightarrow 1$ in (21), we recover the results of [10] as a special case.

Theorem 2.5. Let $p \in \mathbb{N}, k>0, a>1, \frac{1}{a}+\frac{1}{b}=1$ and $m, n \in \mathbb{N}$ such that $\frac{m}{a}+\frac{n}{b} \in \mathbb{N}$. Then, the inequality

$$
\left|\psi_{p, k}^{\left(\frac{m}{a}+\frac{n}{b}\right)}\left(\frac{x}{a}+\frac{y}{b}\right)\right| \leq\left|\psi_{p, k}^{(m)}(x)\right|^{\frac{1}{a}}\left|\psi_{p, k}^{(n)}(y)\right|^{\frac{1}{b}}
$$

holds for $x>0$ and $y>0$.

Proof. From the integral representation (9), we obtain

$$
\begin{aligned}
\left|\psi_{p, k}^{\left(\frac{m}{a}+\frac{n}{b}\right)}\left(\frac{x}{a}+\frac{y}{b}\right)\right| & =\int_{0}^{\infty}\left(\frac{1-e^{-k(p+1) t}}{1-e^{-k t}}\right) t^{\frac{m}{a}+\frac{n}{b}} e^{-\left(\frac{x}{a}+\frac{y}{b}\right) t} d t \\
& =\int_{0}^{\infty}\left(\frac{1-e^{-k(p+1) t}}{1-e^{-k t}}\right)^{\frac{1}{a}+\frac{1}{b}} t^{\frac{m}{a}+\frac{n}{b}} e^{-\left(\frac{x}{a}+\frac{y}{b}\right) t} d t \\
& =\int_{0}^{\infty}\left(\frac{1-e^{-k(p+1) t}}{1-e^{-k t}}\right)^{\frac{1}{a}} t^{\frac{m}{a}} e^{-\frac{x t}{a}} \cdot\left(\frac{1-e^{-k(p+1) t}}{1-e^{-k t}}\right)^{\frac{1}{b}} t^{\frac{n}{b}} e^{-\frac{y t}{b}} d t \\
\leq & {\left[\int_{0}^{\infty}\left(\frac{1-e^{-k(p+1) t}}{1-e^{-k t}}\right) t^{m} e^{-x t} d t\right]^{\frac{1}{a}} } \\
& \times\left[\int_{0}^{\infty}\left(\frac{1-e^{-k(p+1) t}}{1-e^{-k t}}\right) t^{n} e^{-y t} d t\right]^{\frac{1}{b}} \\
= & \left|\psi_{p, k}^{(m)}(x)\right|^{\frac{1}{a}}\left|\psi_{p, k}^{(n)}(y)\right|^{\frac{1}{b}}
\end{aligned}
$$

which concludes the proof.

Note: The absolute signs in (22) are not required if $m$ and $n$ are positive odd integers such that $\frac{m+1}{a}, \frac{n+1}{b} \in \mathbb{N}$.

Corollary 2.3. Let $p \in \mathbb{N}, k>0$ and $m \in \mathbb{N}$. Then the inequality

$$
\left|\psi_{p, k}^{(m)}(x)\right|\left|\psi_{p, k}^{(m+2)}(x)\right|-\left|\psi_{p, k}^{(m+1)}(x)\right|^{2} \geq 0
$$

holds for $x>0$.

Proof. This follows from Theorem 2.5 by letting $x=y, a=b=2$ and $n=m+2$.

Remark 2.5. By letting $p \rightarrow \infty$ in Theorem 2.5, we obtain the $k$-analogue of (22). Also, by letting $k \rightarrow 1$ in Theorem 2.5, we obtain the p-analogue of (22) as presented in Theorem 2.1 of [5].

Remark 2.6. By letting $p \rightarrow \infty$ as $k \rightarrow 1$ in Theorem 2.5, we obtain Theorem 2.5 of [11] as a special case.

Remark 2.7. Let $x=y$ and $a=b=2$ in Theorem 2.5. Then, by letting $p \rightarrow \infty$ as $k \rightarrow 1$, we obtain Theorem 2.1 of [4]. 
Theorem 2.6. Let $m, n, p \in \mathbb{N}$ and $k>0$. Then, the inequality

$$
\left[\left|\psi_{p, k}^{(m)}(x)\right|+\left|\psi_{p, k}^{(n)}(y)\right|\right]^{\frac{1}{u}} \leq\left|\psi_{p, k}^{(m)}(x)\right|^{\frac{1}{u}}+\left|\psi_{p, k}^{(n)}(y)\right|^{\frac{1}{u}}
$$

holds for $x>0$ and $y>0$, where $u$ is a positive integer.

Proof. We employ the Minkowski's inequality for finite sums, and the fact that $a^{u}+b^{u} \leq$ $(a+b)^{u}$, for $a, b \geq 0$ and $u$ a positive integer. From (8), we obtain

$$
\begin{aligned}
{\left[\left|\psi_{p, k}^{(m)}(x)\right|+\left|\psi_{p, k}^{(n)}(y)\right|\right]^{\frac{1}{u}} } & =\left[\sum_{i=0}^{p} \frac{m !}{(i k+x)^{m+1}}+\sum_{i=0}^{p} \frac{n !}{(i k+y)^{n+1}}\right]^{\frac{1}{u}} \\
& =\left[\sum_{i=0}^{p}\left(\left(\frac{(m !)^{\frac{1}{u}}}{(i k+x)^{\frac{m+1}{u}}}\right)^{u}+\left(\frac{(n !)^{\frac{1}{u}}}{(i k+y)^{\frac{n+1}{u}}}\right)^{u}\right)\right]^{\frac{1}{u}} \\
& \leq\left[\sum_{i=0}^{p}\left(\left(\frac{(m !)^{\frac{1}{u}}}{(i k+x)^{\frac{m+1}{u}}}\right)+\left(\frac{(n !)^{\frac{1}{u}}}{(i k+y)^{\frac{n+1}{u}}}\right)\right)^{u}\right]^{\frac{1}{u}} \\
& \leq\left[\sum_{i=0}^{p}\left(\frac{(m !)^{\frac{1}{u}}}{(i k+x)^{\frac{m+1}{u}}}\right)^{u}\right]^{\frac{1}{u}}+\left[\sum_{i=0}^{p}\left(\frac{(n !)^{\frac{1}{u}}}{(i k+y)^{\frac{n+1}{u}}}\right)^{u}\right]^{\frac{1}{u}} \\
& =\left|\psi_{p, k}^{(m)}(x)\right|^{\frac{1}{u}}+\left|\psi_{p, k}^{(n)}(y)\right|^{\frac{1}{u}}
\end{aligned}
$$

which concludes the proof.

Note: The absolute signs in (23) are not required if $m$ and $n$ are positive odd integers.

Remark 2.8. By letting $p \rightarrow \infty$ in Theorems 2.5 and 2.6, we obtain the $k$-analogues of (22) and (23).

Remark 2.9. By letting $k \rightarrow 1$ in Theorems 2.5 and 2.6, we obtain the p-analogues of (22) and (23) as presented in [5].

Remark 2.10. The q-analogues, $(p, q)$-analogues and $(q, k)$-analogues of the inequalities (22) and (23) can be found in [12], [6] and [7] respectively.

Theorem 2.7. Let $p \in \mathbb{N}, k>0$ and $m \in \mathbb{N}$. Then, the inequalities

$$
\begin{aligned}
& \left(\exp \psi_{p, k}^{(m)}(x)\right)^{2} \geq \exp \psi_{p, k}^{(m+1)}(x) \cdot \exp \psi_{p, k}^{(m-1)}(x), \quad \text { if } m \text { is odd } \\
& \left(\exp \psi_{p, k}^{(m)}(x)\right)^{2} \leq \exp \psi_{p, k}^{(m+1)}(x) \cdot \exp \psi_{p, k}^{(m-1)}(x), \quad \text { if } m \text { is even }
\end{aligned}
$$

are satisfied for $x>0$.

Proof. By relation (8), we obtain

$$
\psi_{p, k}^{(m)}(x)-\frac{1}{2}\left[\psi_{p, k}^{(m+1)}(x)+\psi_{p, k}^{(m-1)}(x)\right]
$$




$$
\begin{aligned}
& =\sum_{n=0}^{p} \frac{(-1)^{m+1} m !}{(n k+x)^{m+1}}-\frac{1}{2} \sum_{n=0}^{p} \frac{(-1)^{m+2}(m+1) !}{(n k+x)^{m+2}}-\frac{1}{2} \sum_{n=0}^{p} \frac{(-1)^{m}(m-1) !}{(n k+x)^{m}} \\
& =\frac{(-1)^{m}}{2}\left[2 \sum_{n=0}^{p} \frac{-m !}{(n k+x)^{m+1}}-\sum_{n=0}^{p} \frac{(m+1) !}{(n k+x)^{m+2}}-\sum_{n=0}^{p} \frac{(m-1) !}{(n k+x)^{m}}\right] \\
& =\frac{(-1)^{m+1}}{2}\left[\sum_{n=0}^{p} \frac{2 m !}{(n k+x)^{m+1}}+\sum_{n=0}^{p} \frac{(m+1) !}{(n k+x)^{m+2}}+\sum_{n=0}^{p} \frac{(m-1) !}{(n k+x)^{m}}\right] \\
& =\frac{(-1)^{m+1}}{2} \sum_{n=0}^{p} \frac{(m-1) !}{(n k+x)^{m}}\left[\frac{2 m}{n k+x}+\frac{(m+1) m}{(n k+x)^{2}}+1\right] \\
& =\frac{(-1)^{m+1}}{2} \sum_{n=0}^{p} \frac{(m-1) !}{(n k+x)^{m+2}}\left[(m+n k+x)^{2}+m\right] \\
& =\left\{\begin{array}{l}
\geq 0, \quad m \text { odd } \\
\leq 0, \quad m \text { even. }
\end{array}\right.
\end{aligned}
$$

That implies,

$$
2 \psi_{p, k}^{(m)}(x) \geq \psi_{p, k}^{(m+1)}(x)+\psi_{p, k}^{(m-1)}(x)
$$

and

$$
2 \psi_{p, k}^{(m)}(x) \leq \psi_{p, k}^{(m+1)}(x)+\psi_{p, k}^{(m-1)}(x)
$$

respectively for odd $m$ and even $m$. Then, by exponentiating the inequalities (26) and (27), we obtain the desired results.

Remark 2.11. By letting $p \rightarrow \infty$ in Theorem 2.7, we obtain the $k$-analogues of (24) and (25).

Remark 2.12. By letting $k \rightarrow 1$ in Theorem 2.7, we obtain the p-analogues of (24) and (25) as presented in Theorem 2.5 of [5] as a special case.

Remark 2.13. By letting $p \rightarrow \infty$ as $k \rightarrow 1$ in Theorem 2.7, we obtain Theorem 3.2 of [8] as a special case.

\section{Conclusion}

We have introduced a new two-parameter deformation of the classical Gamma function, called the $(p, k)$-analogue. In addition, we have established some identities and inequalities involving this new function. The established results provide generalizations of some known results in the literature.

\section{Acknowledgements}

The authors are very grateful to the anonymous referees for their useful comments and suggestions, which helped in improving the quality of this paper. 


\section{References}

[1] T. M. Apostol, Introduction to Analytic Number Theory, Springer-Verlag, 1976.

[2] R. Díaz and E. Pariguan, On hypergeometric functions and Pachhammer $k$-symbol, Divulgaciones Matemtícas, 15(2)(2007), 179-192.

[3] C. G. Kokologiannaki, Some Properties of $\Gamma_{q, k}(t)$ and Related Functions, International Journal of Contemporary Mathematical Sciences, 11(1)(2016), 1-8.

[4] A. Laforgia and P. Natalini, Turan type inequalities for some special functions, J. Ineq. Pure Appl. Math., 7(1)(2006), Art. 22, 1-5.

[5] F. Merovci, Turan type inequalities for p-polygamma functions, Le Matematiche, Fasc II (2013), 99-106.

[6] F. Merovci, Turan type inequalities for (p, q)-Gamma function, Scientia Magna, 9(1) (2013), 2530.

[7] F. Merovci, Turan type inequalities for some $(q, k)$-Special functions, Acta Universitatis Apulensis, 34 (2013), 69-76.

[8] C. Mortici, Turan type inequalities for the Gamma and Polygamma functions, Acta Universitatis Apulensis, 23 (2010), 117-121.

[9] E. Neuman, Inequalities involving a logarithmically convex function and their applications to special functions, J. Inequal. Pure Appl. Math., 7(1)(2006) Art. 16.

[10] J. Sándor, A note on certain inequalities for the gamma function, J. Ineq. Pure Appl. Math., 6(3)(2005), Art. 61.

[11] W. T. Sulaiman, Turan inequalities for the digamma and polygamma functions, South Asian Journal of Mathematics, 1(2)(2011), 49-55.

[12] W. T. Sulaiman, Turan type inequalities for some special functions, The Australian Journal of Mathematical Analysis and Applications, 9(1)(2012), 1-7. 